\title{
La Guerra de Corea y la República Popular China: una aproximación constructivista ${ }^{1}$
}

\author{
Korean War and Popular Republic China: \\ A constructivist approach
}

DOI: $10.32870 /$ mycp.v9i25.607

Daniel Lemus-Delgado²

\begin{abstract}
Resumen
El objetivo de este artículo es analizar la política exterior de China en la Guerra de Corea basado en un enfoque teórico constructivista. A partir de la investigación documental, este artículo analiza la relación entre las identidades e intereses de China que le llevaron a la élite política china a involucrarse en este conflicto armado. Como resultado de la investigación, este artículo destaca cómo la relación mutuamente constitutiva entre estructuras materiales y no materiales se refleja en la reformulación de las identidades y su impacto en el escenario internacional. De esta manera, este artículo aporta a los análisis de política exterior, el hecho de que en la toma de decisiones de los líderes interviene también la identidad.
\end{abstract}

Palabras claves: Constructivismo, Identidad Nacional, Guerra de Corea, China, Estados Unidos.

\begin{abstract}
This article aims to analyze the Foreign Policy of China in the Korean War based on a constructivist theoretical approach. So, I analyze the relationship between China's identities and interests and its impact on the Chinese political elite to decide their participation in the Korean War. I employ documentary research to examine the relationship between China's identities and interests and its impact on the Chinese political elite. As a result of the research, I conclude how the reformulation of identities reflects the mutually constitutive relationship between material and non-material structures. In this way, this article provides some elements to understand how in the decision-making of Foreign Policy the identity also influences the decisions and actions of the leaders.
\end{abstract}

Key Words: Constructivism, National Identities, Korean War, China, The United States.

Artículo recibido el 06 de noviembre de 2018 y dictaminado el 07 de junio de 2019.

1. El autor agradece el apoyo recibido por parte de la Universidad de Fudan para la realización de este artículo en el marco del programa Visiting Scholar auspiciado por Fudan Development Institute.

2. Instituto Tecnológico y de Estudios Superiores de Monterrey (ITESM), Escuela de Ciencias Sociales y Gobierno. Av. Gral. Ramón Corona 2514, Zapopan, Jalisco, México. CP 45201. ORCID: https:// orcid.org/0000-0003-1002-5319. Correo electrónico: dlemus@tec.mx 


\section{Introducción}

El fin de la Segunda Guerra Mundial y la rendición incondicional de Japón impactó profundamente en el equilibrio de poderes en el Este de Asia. A su vez, permitió la liberación de Corea, territorio que dejó ser una colonia del imperio japonés. Sin embargo, este hecho fue acompañado por una enorme tragedia para el pueblo coreano, la división de su nación como consecuencia de una disputa ideológica que se originó en el contexto de la Guerra Fría. Paradójicamente, la Guerra de Corea fue, ante todo, el deseo por unificar una nación. Este conflicto armado no solamente marcó a la historia de Corea, sino tuvo un impacto profundo en el equilibrio de poderes en la región.

El caso de China es significativo porque su involucramiento en la guerra sentó las bases para la nueva imagen que este país proyectó en el escenario internacional en los años posteriores al conflicto. A pesar del enorme costo que China pagó tanto en recursos humanos como en recursos materiales, el rol que China desempeñó en este conflicto le permitió presentarse en los años posteriores como un país líder de una tercera opción, más allá del bloque capitalista encabezado por los Estados Unidos y el bloque soviético liderado por la Unión Soviética.

A partir de la Guerra de Corea, este artículo analiza la manera en que, en el escenario internacional, los Estados se comportan motivados no solamente por su deseo de alcanzar sus intereses materiales. Las aspiraciones no materiales, como es la búsqueda de prestigio y el fortalecimiento de una identidad nacional, son igual de importantes. Basado en el enfoque teórico del constructivismo que enfatiza que los intereses e identidades de los actores son socialmente construidos (Philips, 2007), este artículo analiza los antecedentes de la Guerra de Corea, el contexto histórico en que China se involucró en este conflicto y las consecuencias que tuvo para este país.

El constructivismo sostiene que la realidad es construida socialmente y no nos es dada por naturaleza (Chernoff, 2007). El mundo que tenemos es consecuencia de nuestras interacciones sociales y no es el resultado de estructuras universales, eternas, inmutables; en otras palabras, el mundo en que vivimos es resultado "de nuestra hechura" (Onuf, 1998). Incluso, los temas más espinosos como la anarquía del sistema internacional, para los constructivistas no es más que un constructo social (Wendt, 1992). Desde el enfoque teórico constructivista, este artículo explica en un caso histórico concreto la 
manera en que las identidades de los Estados influyen y son influidas por las estructuras materiales en que estos se desempeñan.

Desde esta perspectiva, el argumento central de este artículo es que las identidades importan cuando se trata de comprender el actuar de los Estados en el escenario internacional. Como lo muestra el involucramiento de China en la Guerra de Corea, la identidad del Estado chino fue un factor fundamental que influyó en los líderes políticos para inmiscuirse en una guerra de la que se tenía conciencia que sería costosa y no necesariamente corta. Al mismo tiempo, los acontecimientos y consecuencias de la guerra, embebidas en las estructuras materiales pero dotadas de significados compartidos construidos socialmente, reformularon la identidad del Estado chino en los siguientes años.

Este artículo está organizado en cinco secciones. En la primera se presenta sucintamente las bases del enfoque teórico del constructivismo enfatizando su contribución a comprender el rol de las identidades de los Estados en el escenario internacional. La segunda sección comprende los antecedentes de Guerra de Corea con la intención de presentar una visión panorámica del contexto histórico que condicionó el involucramiento de China. En la siguiente sección se observan las generalidades del conflicto armado hasta el ingreso de China en la guerra. Posteriormente, se analiza el dilema que enfrentó la élite política de China sobre la conveniencia de ir a la guerra. Más adelante se analizan las consecuencias para China en la Guerra de Corea. Finalmente, este artículo concluye con una reflexión sobre cómo, si se consideran las identidades de los Estados, nuestras explicaciones sobre la dinámica de los asuntos internacionales pueden ser más robustas.

\section{Identidad e intereses, una aproximación constructivista}

El constructivismo es un enfoque teórico social que propone comprender los asuntos internacionales desde una perspectiva más vasta que las teorías tradicionales, pues afirma que las relaciones internacionales existen dentro de un conjunto más amplio de interacciones sociales y políticas. La manera en que se presentan estas relaciones determinan cómo los Estados actúan en esa realidad a la que llamamos escenario internacional (Kaufman, 2013).

El mundo social y político, conforme al constructivismo, no es una realidad externa y objetiva que se encuentre más allá de la conciencia humana. Por lo tanto, no existe un aislamiento temporal de los asuntos internacionales. Las relaciones entre los Estados existen como conciencia intersubjetiva; es una 
invención o creación humana no de tipo físico o material, sino intelectual e ideológica. Por lo tanto, el escenario internacional en que cada Estado desempeña un rol específico es un conjunto de ideas y un sistema de normas, que ha sido organizado por ciertas personas en un momento y lugar determinado (Jackson \& Sorensen, 2003).

Conforme a Wendt (1994), el constructivismo se basa en tres afirmaciones elementales. Primero, los Estados son las principales unidades de análisis de la teoría política internacional. Segundo, las estructuras fundamentales en el sistema estatal son intersubjetivas, en lugar de ser exclusivamente materiales. Tercero, las identidades e intereses de los Estados son construidas por las estructuras sociales, en lugar de ser exógenamente dadas al sistema por la naturaleza humana o por la política doméstica. Así, los Estados forman ideas y entendimientos del mundo que los rodea, basándose en las estructuras en las que interactúan y actúan posteriormente con base en las percepciones que forman a partir de estas interacciones (Wendt, 1994).

De esta manera, el constructivismo propone superar la estrecha visión del realismo que destaca que los Estados actúan en el escenario internacional de manera lógica, calculadora y racional, conforme a sus capacidades de poder (Hobson, 2000). Al contrario, el constructivismo señala que en los intereses de los Estados tienen la misma importancia las estructuras materiales que las estructuras normativas, las cuales son constituidas desde el mundo de las ideas (Reus-Smith, 2005). De hecho, los elementos materiales solo adquieren significado para la acción humana a través de la estructura de conocimiento compartido, el cual está socialmente embebido (Adler, 2002). De esta manera, los Estados son forzados a actuar no solo por causas materiales, sino también por las estructuras sociales normativas que prevalecen en el contexto internacional (Hobson, 2000). Por lo tanto, la manera que el Estado cumple sus metas y se relaciona con otros ante todo es una cuestión social (Griffiths, O'Callaghan, \& Roach, 2008). En estas interacciones, las identidades son necesarias para garantizar al menos un nivel mínimo de previsibilidad y orden en el sistema internacional (Hopf, 1998).

Para el constructivismo, el comportamiento de los Estados es explicado esencialmente como el resultado de las normas. Así, los Estados y otros agentes del escenario internacional, buscan garantizar que exista una correspondencia entre su propia conducta y las normas interiorizadas que provee de legitimidad a sus acciones. Además, estas normas se derivan de sus propias identidades. Por lo tanto, normas e identidades se influyen mutuamente. 
De esta manera, lejos de ser puramente decorativas, las normas ejercen una profunda influencia en el comportamiento del Estado tanto ayudando a constituir identidades como conformando los intereses de los Estados. Pero también, las normas condicionan y restringen las estrategias y acciones de los Estados en el cumplimiento de estos intereses. Esta posición contrasta con el supuesto racionalista de que el comportamiento de los agentes se rige simplemente por una lógica de las consecuencias.

Ahora bien, al aceptar que las normas sociales son un elemento fundamental que determina el quehacer de los Estados, el constructivismo considera también que las ideas, las creencias y las identidades colectivas son la clave para comprender el escenario internacional (Genest, 2004). En consecuencia, la identidad afecta las preferencias y las metas de Estados en el escenario internacional (Chernoff, 2007). Respecto a la pregunta sobre cuáles eran los intereses fundamentales de China al involucrarse en la Guerra de Corea, la respuesta desde el constructivismo es que dichos intereses fueron determinados socialmente por medio de una interpretación del mundo. Como lo muestra el caso de China en la Guerra de Corea, la interpretación del mundo es al mismo tiempo dinámica y colectiva. Además, esta interpretación es el resultado de una interacción permanente entre el Estado y otros agentes en el escenario internacional en donde la identidad juega un papel clave.

Así, desde el constructivismo la clave para comprender la relación entre identidad nacional y la política exterior se encuentra en la construcción social de la realidad, lo cual comprende también los fundamentos de la identidad nacional (Kowert, 1998). Alexander Wendt (1992) sugiere que los actores del sistema internacional adquieren identidades al participar en significados colectivos. Las identidades, en palabras de Wendt, son los “...entendimientos específicos del rol y expectativas acerca del yo, relativamente estables" (Wendt, 1992, p. 397). Por lo tanto, se puede afirmar que la identidad nacional es una realidad política y social construida en contextos históricos y sociales específicos, la cual está sujeta a cambios y reconfiguraciones, especialmente bajo la movilización intensiva de las élites en épocas de transición de regímenes y/o restructuración mundial (Chu \& Lin, 2003).

Las identidades implican un conjunto particular de intereses o preferencias con respecto a las opciones de acción en particular. Un Estado comprende a otros de acuerdo con la identidad que les atribuye, mientras que al mismo tiempo reproduce su propia identidad a través de la práctica social diaria (Hopf, 1998). Los Estados, al igual que las personas, pueden tener identida- 
des múltiples (Kaufman, 2013). De esta manera, los Estados responden a las acciones de otros Estados dependiendo, en parte, de cómo el Estado se ve a sí mismo, así como las formas en que ve a los demás Estados. Evidentemente, en la medida en que cambian las circunstancias, las identidades cambian a través del tiempo dependiendo de las interacciones entre esos Estados y las formas en que se perciben a sí mismos (Kaufman, 2013). Es crucial observar que quienes crean y reproducen sus propias identidades no tienen el control de lo que finalmente esa identidad significará para los demás, lo que Hopf (1998) denomina la estructura intersubjetiva como el árbitro final del significado.

Finalmente, los constructivistas sugieren que los intereses son también el producto de la identidad. Por ejemplo, cuando se afirma que un Estado posee la identidad de una potencia mundial implica que posee un conjunto particular de intereses diferentes a los implícitos en la identidad de una potencia emergente. De este modo, al convertir los intereses en una variable central, el constructivismo explora no solo cómo se desarrollan los intereses particulares sino sus significados con relación a su identidad (Hopf, 1998).

Estos supuestos teóricos constructivistas se reflejan en la intervención de China en la Guerra de Corea, ya que los intereses detrás de sus acciones estuvieron en parte determinados por la identidad que en ese momento China tenía, un país autopercibido como una víctima de las ambiciones imperialistas de las potencias extranjeras. A su vez, el participar en este conflicto permitió modificar su identidad al grado tal de proyectarse posteriormente como una nación líder de un tercer grupo de países que no pertenecían ni al bloque capitalista ni al socialista. En la siguiente sección se exploran las circunstancias de la división de Corea con la intención de comprender el contexto histórico en que se reformuló la identidad del Estado chino.

\section{La división de Corea}

La Conferencia de El Cairo fue el antecedente inmediato a la división de Corea y el posterior conflicto armado cuyo principal objetivo fue la unificación del país. En esta reunión, realizada en el año de 1943, participaron el Presidente de los Estados Unidos, Franklin Roosevelt, el Primer Ministro Británico, Winston Churchill y el Presidente de la República de China, Chiang Kai-shek (Robinson, 2007). La Declaración de El Cairo, resultado de este encuentro, fue el primer pronunciamiento público sobre lo que los países aliados planeaban para el futuro de Corea una vez que finalizara el conflicto armado. Para en- 
tonces, Corea no era de gran interés o preocupación para los Estados Unidos o la Gran Bretaña; sin embargo, Roosevelt tenía la idea de que Corea debía colocarse bajo un tutelaje por parte de los países vencedores. Probablemente, esta manera de pensar pudo haber sido influenciada por el rol de los Estados Unidos en las Filipinas, en donde los estadunidenses consideraban que preparaban a esa colonia para su independencia completa, la cual fue prevista para 1946 (Seth, 2010).

La decisión de dividir a Corea no se basó en ningún conocimiento real de Corea ni de su historia, su cultura o las fuertes aspiraciones nacionalistas de su pueblo. Más bien, la división de Corea fue el producto de la conveniencia o comodidad de los aliados (Seth, 2010). El paralelo 38 fue una línea arbitraria en el mapa y no correspondía a ninguna división geográfica, cultural o histórica del país (Robinson, 2007). Corea había sido un país unificado desde el siglo VII (Murphey, 2009). La formación de un sentimiento nacional bajo la sombra del dominio japonés provocó que ningún coreano aspirara a la división permanente de Corea en dos Estados distintos (Kwon, 2016). Al igual que en otras partes del imperio japonés, el sorpresivo anuncio en la radio del emperador Hirohito anunciando la rendición de Japón fue para la mayoría de los coreanos una tremenda sacudida (Kawamura, 2015). Al enterarse de la derrota del Japón, prácticamente todos los coreanos pensaron que la independencia era inminente sin sospechar de los planes de las potencias aliadas sobre el futuro de la nación (Seth, 2010).

Las potencias aliadas se reunieron en la Conferencia de Moscú en diciembre de 1945 para discutir los problemas de la posguerra que incluían la delicada situación de Corea. El acuerdo al que se llegó en Moscú preveía la creación de un gobierno democrático provisional coreano, el establecimiento de una comisión mixta de las dos potencias ocupantes y la preparación para la tutela de cuatro poderes de Corea por un período hasta por cinco años (Lee, 2001). Sin embargo, este acuerdo sería un serio obstáculo para la futura unificación de Corea en la medida en que se extendió la desconfianza entre los Estados Unidos y la Unión Soviética y se extendiera la rivalidad derivada de la Guerra Fría (Manson, 1996).

En Corea, cuando los acuerdos alcanzados en Moscú se hicieron públicos, se desató un malestar generalizado. Los representantes de todas las facciones políticas, incluyendo inicialmente también a los comunistas coreanos, se opusieron a esta resolución porque confirmaba que, conforme a la voluntad de las grandes potencias, Corea no sería inmediatamente un Estado indepen- 
diente (Kleiner, 2001). De esta manera, con el paso del tiempo, los coreanos moderados que se ubicaban en el centro del espectro ideológico y que se negaron a aliarse con cualquiera de los poderes hegemónicos e insistieron en una reunificación pacífica de su país, fueron marginados y, en algunos casos, aterrorizados físicamente, para que se aliaran a uno de los dos bloques extranjeros, fomentando la creación momentánea de dos gobiernos distintos en Corea (Lee, 2006). Como lo ha observado Chan (2018), la evidencia histórica incuestionable ha demostrado que las dos grandes potencias de la época, los Estados Unidos y la Unión Soviética, fueron responsables de crear el caso coreano de "una nación, dos países". De hecho, los líderes políticos de ambos Estados terminaron por alinearse firmemente a una de estas grandes potencias, pero al finalizar la Segunda Guerra Mundial ninguna de estas dos potencias estaba verdaderamente preocupada por la expectativa de los coreanos de restablecer un Estado soberano que pudiera ejercer plenamente la autonomía y la autodeterminación.

Las políticas de ocupación soviéticas y norteamericanas favorecieron la polarización de la política y la aparición de regímenes ideológicamente opuestos. En febrero de 1946, Kim Il-sung formó el Consejo Popular Interino, mientras que en el sur Syngman Rhee fundó el Consejo Representativo, el precursor de la Asamblea Legislativa interina de Corea del Sur (Leitich, 2001). El conflicto ideológico entre el norte y el sur continuó cuando Estados Unidos presentó la cuestión de Corea en la recién creada Naciones Unidas en agosto de 1947; en consecuencia, se estableció la Comisión Temporal de las Naciones Unidas para Corea (CTNUC). El plan de las Naciones Unidas era llevar a cabo elecciones en toda Corea para establecer una Asamblea Nacional. De esta manera, el poder sería transferido a las nuevas autoridades políticas, las fuerzas soviéticas y norteamericanas se retirarían de la península y Corea alcanzaría su plena soberanía (Lee, 2001).

La Unión Soviética no reconoció la autoridad de la CTNUC y no impulsó la celebración de elecciones en el norte (Kleiner, 2001). En el sur, las elecciones patrocinadas por las Naciones Unidas se llevaron a cabo entre mayo y agosto de 1948 (Seth, 2010). Una vez concluido el ejercicio Syngman Rhee declaró a la República de Corea afirmando ser el único gobierno legítimo de Corea (Leitich, 2001). En el norte, sin la intervención de las Naciones Unidas, se celebraron elecciones y se proclamó la República Popular Democrática de Corea en el mes de septiembre (Leitich, 2001). A partir de entonces, Corea tuvo dos gobiernos, cada uno afirmando su derecho a gobernar sobre toda la 
península coreana. Después de las elecciones, tanto los soviéticos como los norteamericanos se retiraron de sus zonas de ocupación, estableciendo el escenario para la Guerra de Corea (Robinson, 2007).

A diferencia de la ordenada y bien organizada ocupación soviética en el norte, la ocupación norteamericana estuvo marcada por la confusión, la falta de preparación y planificación (Seth, 2010). La República de Corea inició su existencia con un Estado caótico y precario (Oberdorfer \& Carlin, 2014). El Presidente Syngman Rhee gobernó de manera autoritaria, basado en su alianza con las élites sociales y económicas conservadoras; haciendo uso de un amplio aparato represivo soportado por la policía nacional y contando con el respaldo creciente de los militares (Robinson, 2007). Los Estados Unidos apoyaron a Rhee proporcionando capacitación y asesoría militar, asistencia económica y, lo que es más importante, un contexto legitimador para justificar la represión bajo el argumento de una lucha contra el comunismo que culminó con el establecimiento de la República de Corea en 1948 (Painter, 1999).

Por su parte, Corea del Norte entre 1948 - 1950 experimentó un periodo de gran estabilidad (Oberdorfer \& Carlin, 2014). El nuevo gobierno se encontraba firmemente al mando e iniciaron reformas radicales. El régimen lanzó programas masivos para promover la alfabetización de los adultos (Seth, 2010). Además, se nacionalizaron las principales industrias y se puso en marcha el desarrollo de una economía viable bajo un plan económico, diseñado por economistas educados en Japón (Kleiner, 2001). Asimismo, Corea del Norte se benefició de la variedad de fábricas japonesas nacionalizadas, así como los ricos recursos minerales con los que contaba y sus vastas fuentes de energía eléctrica generadas por las represas hidroeléctricas construidas durante el periodo colonial (Seth, 2010).

Pero lo más importante fue la reforma agraria que se había llevado a cabo desde 1946, en la que se confiscaron grandes extensiones de tierra, las cuales se redistribuyeron entre los agricultores arrendatarios y los pequeños productores (Kim, 2012). Las autoridades confiscaron y redistribuyeron todas las propiedades agrícolas que habían pertenecido a los japoneses y las de cinco mil terratenientes coreanos. Aquellos terratenientes que no fueron considerados colaboradores directos de las autoridades japonesas durante el periodo de ocupación se les permitió mantener una pequeña porción de tierra, siempre y cuando ellos mismos la cultivaran (Robinson, 2007). Esta fue una revolución en la sociedad coreana porque atacó el fundamento mismo de la distribución inequitativa de la riqueza que había apoyado a la clase dirigente 
en Corea durante siglos (Robinson, 2007). Esto proporcionó una base para el apoyo rural y ayudó a legitimar el nuevo Estado (Seth, 2010).

\section{El conflicto armado}

La compleja situación en la península coreana empeoró en la medida que avanzó la Guerra Fría. La rivalidad entre Estados Unidos y la Unión Soviética ya existía en 1945, pero se intensificó durante los siguientes años. En el centro de la Guerra Fría estaba el miedo mutuamente percibido de un posible ataque sorpresa por el otro bando, un temor que fue alimentado por mutuas percepciones erróneas y una falta de comprensión entre sí (Hughes \& Dockrill, 2006). El apoyo de la Unión Soviética a los movimientos comunistas internacionales embebido por una visión de la historia mundial que presentaba como inevitable la lucha entre el mundo socialista y capitalista determinaron las relaciones internacionales durante más de cuatro décadas (Painter, 1999). La Guerra Fría, evidenciada en la doctrina Truman proclamada en marzo de 1947 que contemplaba la política americana de contención, tuvo un impacto fundamental en los sucesos que se presentaron en la península de Corea (Krieger, 2006).

Una gran controversia ha sido el origen de la guerra. Como lo ha señalado Seth (2010), la Guerra ha sido considerada como inevitable por algunos, un error trágico y evitable por otros. Lo cierto es que la escalada del conflicto que derivó en la Guerra de Corea se originó fundamentalmente del enfrentamiento de dos ideologías opuestas compitiendo por su supremacía en la península (Simons, 1995). Los líderes de las dos Coreas consideraron que el establecimiento de regímenes separados era sólo temporal; tanto Syngman Rhee como Kim Il-sung alentaron en sus territorios la sensación de una pronta reunificación por todos los medios posibles, claro que bajo su propio control (Beal, 2011). A lo largo del paralelo 38 antes del inicio de la Guerra ocurrieron enfrentamientos frecuentes entre las dos Coreas (Sandler, 1999).

En los años de posguerra Asia fue un área de creciente interés geoestratégico para los Estados Unidos (Buckley, 2007). La Guerra Civil en China, de 1946 a 1949, terminó con la victoria comunista y la proclamación de la República Popular el 1 de octubre de 1949 (Murphey, 2009). En febrero de 1950, Mao Zedong firmó el Tratado de Amistad, Alianza y Asistencia Mutua con la Unión Soviética (Chen, 2001). Los Estados Unidos, que habían invertido considerable ayuda militar para apoyar a los nacionalistas bajo Chiang Kai- 
shek, habían fracasado en su apoyo (Buckley, 2007). La "pérdida" de China presionó a la administración Truman para que dibujara la línea de contención en Asia, aunque para entonces la mayoría de los políticos estadunidenses deseaban evitar una guerra terrestre en este continente (Painter, 1999). Sin embargo, los Estados Unidos no tenían claridad acerca de la magnitud de su compromiso en el Este de Asia. El ejemplo más famoso es el discurso - aprobado por el presidente Truman- del Secretario de Estado, Dean Acheson en el que estableció lo que él llamó el "perímetro de defensa americano" en el Pacífico, en el cual Corea quedaba claramente fuera (Simons, 1995).

A su vez, inicialmente la Unión Soviética también trató de limitar su ayuda a Corea y se mostró reacio a apoyar una guerra (Seth, 2010). Sin embargo, esta postura cambió con el transcurso del tiempo en la medida en que Stalin, a partir de los acontecimientos ocurridos a finales de la década de 1940, consideró que la situación internacional había cambiado lo suficiente como para brindar un mayor apoyo al régimen norcoreano (Miller \& Wich, 2011). A pesar de que las fuerzas soviéticas se retiraron al final de 1948, la Unión Soviética proporcionó equipos militares como tanques y artillería al nuevo gobierno mientras que miles de soldados coreanos fueron enviados a la URSS para entrenarse en el uso de este equipo (Oberdorfer \& Carlin, 2014).

Pero el factor clave en la acumulación militar de Corea del Norte fue la determinación de su liderazgo, especialmente Kim Il-sung, para unificar el país por la fuerza y su insistencia por obtener el apoyo de la Unión Soviética, primero, y de la República Popular China, después (Armstrong, 2013). Los líderes norcoreanos pusieron sus esperanzas en que el inevitable conflicto armado sería apoyado en parte por las guerrillas comunistas en el sur que habían sido duramente golpeadas por una represión sin cuartel por parte del gobierno de Rhee que, para abril de 1950, había asesinado a 36,000 personas y desplazado a 432,000 (Simmons, 1995).

El ejército de Corea del Norte contaba con 150,000 hombres en 1950, en comparación con los menos de 100,000 hombres de la República de Corea (Seth, 2010). Además, Corea del Norte contaba con un ejército más experimentado como consecuencia de los soldados enviados a Manchuria para apoyar a los comunistas chinos durante la Guerra Civil china (Hexiu, 2015). Con la victoria comunista en 1949, decenas de miles de veteranos empezaron a regresar a Corea del Norte (Seth, 2010). Con sus tanques, artillería y otros armamentos, así como con tropas curtidas en el combate, Kim estaba convencido de que cualquier invasión del sur sería fácil y en cuestión de pocos días 
podía conquistar todo el territorio coreano (Kleiner, 2001). A estos hechos es preciso sumar que la capital, Seúl, no estaba lejos de la frontera y se tenía la expectativa que la ciudad se rendiría poco después de la invasión. Finalmente, la dirigencia norcoreana pensaba que la República de Corea se derrumbaría antes de que los norteamericanos pudieran intervenir (Armstrong, 2013).

Kim y otros dirigentes norcoreanos visitaron Moscú entre 1949 y 1950 para persuadir a Stalin de apoyar una invasión. Al principio, Stalin se mostró reacio a involucrarse, pero los líderes de Corea del Norte lograron convencerlo de que ésta era una victoria segura. A Stalin le pareció una buena idea unificar a Corea para que sirviera como un Estado "colchón" en su frontera (Miller \& Wich, 2011). En marzo, Stalin aceptó apoyar el plan de invasión si Mao se comprometía a ayudar si fuera necesario. Después de algunas reservas, Mao acordó apoyar el plan en abril de aquel año. Moscú envió un equipo de expertos militares para ayudar en la elaboración de los planes. Finalmente, Stalin dio el visto bueno final para su aprobación para la invasión (Kleiner, 2001).

La Guerra dio inició el 25 de junio de 1950 (Kim, 2012). El ejército norcoreano se centró inicialmente en capturar Seúl (Leitich, 2001). El plan de Kim era capturar rápidamente la capital. Es posible que él pensara que con esta medida pronto el gobierno surcoreano se desmoronaría (Seth, 2010). Las fuerzas de la República de Corea sólo pudieron defender Seúl durante dos días y luego empezaron a desmoronarse (Leitich, 2001).

El ataque tomó por sorpresa a los Estados Unidos, la República de Corea y la mayor parte del mundo. El 7 de julio, el Consejo de Seguridad de la ONU estableció un comando militar unificado bajo los Estados Unidos con el fin de repeler la agresión norcoreana dirigido por el General MacArthur (Kim, 2012). Eventualmente, dieciséis naciones contribuyeron con fuerzas a esta tarea, más de carácter simbólico que con un peso específico en el desarrollo de las confrontaciones (Seth, 2010). Además, el gran soporte por parte de grupos guerrilleros que Kim esperaba ayudarían en la guerra nunca aparecieron, aunque hubo modestas actividades guerrilleras en las montañas (Robinson, 2007).

En retrospectiva, es posible afirmar que la Guerra de Corea fue, en realidad, tres guerras. De julio a octubre de 1950, Corea del Norte se enfrentó a las fuerzas de la ONU lideradas por los Estados Unidos. La entrada de la China comunista, coincidiendo con las elecciones parlamentarias de mitad de período en los Estados Unidos, constituyó una "nueva" guerra, que duró desde noviembre de 1950 hasta el comienzo de las conversaciones de armisticio en julio de 1951. Los dos últimos años del conflicto constituyeron la 
Tercera Guerra, que fue un sangriento estancamiento en el que las dos partes simplemente trataron de infligir víctimas máximas para obtener una ventaja negociadora en las conversaciones de paz (Blomstedt, 2016).

\section{La decisión de la élite política China para involucrarse en la guerra}

Durante siglos, las relaciones entre China y Corea se rigieron por el modelo del sistema tributario que tenían el objetivo político de garantizar la paz en las fronteras de China (Chan, 2018). Sin embargo, durante el siglo XIX, China experimentó una gradual y constante decadencia que le llevó a vivir amenazada por las potencias internacionales lo que, sumado a sus propias luchas internas, le impidieron reivindicar sus propios intereses respecto a Corea. El Tratado de Shimonoseki, consecuencia de la guerra sino-japonesa firmado en abril de 1895, incluyó la cesión de la península de Liaodong y Taiwán a Japón, así como el reconocimiento formal de independencia de Corea (Wright, 2001). Este reconocimiento abrió la puerta para que posteriormente Japón convirtiera a Corea en una posesión más de su imperio, ocupando su territorio a partir del año de 1910 (Murphey, 2009).

En 1949, al triunfo del comunismo, la tarea de los líderes de la nueva república era titánica. Para entonces, el desempleo y la inflación iban en aumento. Muchos pueblos y ciudades, incluyendo Shanghai y Tianjin, se encontraban en ruinas en gran parte como resultado de los bombardeos del ejército nacionalista; Shanghái contaba con casi 600,000 desempleados, incluidos los refugiados de guerra y los ex soldados del ejército nacionalista, además que más del 70 por ciento de las 12,000 fábricas de la ciudad fueron clausuradas o se encontraban en una situación inoperable (Sun \& Li, 2014). La producción agrícola, que era la base económica, había caído muy por debajo de los niveles anteriores a la Segunda Guerra Mundial (Kennedy, 2011). Entre 1936 y 1949 , las cosechas de granos y de algodón disminuyeron un 22 por ciento y 48 por ciento, respectivamente (Kennedy, 2011). La industria fue golpeada aún más fuerte; la producción industrial total se redujo en un 50 por ciento y la industria pesada en un 70 por ciento (Kennedy, 2011). La guerra había dejado al sistema ferroviario severamente dañado. Por si esta situación fuera poco, aún había que terminar de pacificar el país ya que el nuevo gobierno estimaba que más de 400,000 bandidos deambulaban por China (Kennedy, 2011). Además, la participación de China en la economía mundial había caído 
a 4.5 por ciento a mediados del siglo, en comparación con casi 9 por ciento en 1913 (Kennedy, 2011).

Evidentemente, la política exterior china estaba condicionada por estas circunstancias, a la vez que se esperaba que ésta fuera un instrumento para impulsar la estabilidad y el crecimiento económico. El punto de partida de la política exterior china radicó en la doctrina revolucionaria de los dirigentes comunistas encabezados por Mao. Así, fueron tres principios los que se establecieron como guía para los asuntos internacionales. El primero de estos expresaba la resolución del gobierno para empezar una nueva etapa en la política exterior; por lo tanto, el Partido Comunista rehusó reconocer los vínculos diplomáticos establecidos por el gobierno nacionalista y prefirió establecer relaciones diplomáticas sobre nuevas bases. El segundo principio implicaba que antes de desarrollar nuevos vínculos con otras naciones, el gobierno comunista estaba determinado a expulsar del país todas las fuerzas imperialistas. Finalmente, el tercer principio, conocido como "inclinarse hacia un lado" indicaba que China había decidido adherirse al campo socialista liderado por la Unión Soviética con la intención de concretizar sus intereses nacionales a través de la consolidación y fortalecimiento del socialismo chino (Men, 2007).

En este contexto, desde finales de 1949, los líderes chinos habían considerado inevitable una confrontación con los Estados Unidos; por ejemplo, Zhou Enlai, Premier de la República Popular, difundió el concepto de enfrentar a los Estados Unidos en tres frentes: Taiwán, Vietnam y Corea. (Kleiner, 2001). Por su parte, Mao sospechó que algún día tendría que luchar contra los estadunidenses. Para él, el comunismo internacional era real y el capitalismo era una amenaza también real. Sin embargo, Mao sabía que China no podía luchar sola contra Estados Unidos (Li, 2014). Por lo tanto, tenía que involucrar a los soviéticos. De ahí que una tarea urgente fue buscar una alianza con la Unión Soviética. En diciembre de 1949, Mao visitó Moscú y firmó el Tratado Sino-Soviético de Amistad, Alianza y Asistencia Mutua con Joseph Stalin en febrero del año siguiente (Sun \& Li, 2014).

Los acontecimientos se precipitaron cuando el curso de la guerra cambió abruptamente. Después del arrollador avance del ejército norcoreano, la entrada de Estados Unidos a la guerra cambió radicalmente el equilibrio de fuerzas. En consecuencia, las tropas comandadas por el veterano General Mc Arthur no solamente salvaron el último reducto en manos de las tropas sudcoreanas, sino que repelieron el avance del ejército comunista más allá del 
paralelo 38 (Leitich, 2001). El gobierno del presidente Truman, ignorando los preceptos originales del mandato de las Naciones Unidas, decidió invadir Corea del Norte con la intención de derrotar completamente al régimen de Kim Il-sung (Kim, 2012).

El gobierno estadunidense ignoró las advertencias de China de que intervendría en el conflicto si las tropas de la coalición avanzaban hacia el norte en dirección del río Yalu. Finalmente, el 26 de noviembre de 1950, miles de "voluntarios" comunistas chinos, muchos de ellos pobremente equipados, invadieron Corea. Su presencia empujo a las fuerzas de la ONU hacia el sur, más allá del paralelo 38. El 4 de enero de 1951, las tropas comunistas chinas capturaron Seúl. Sin embargo, para abril de 1951, las fuerzas de la ONU habían llevado nuevamente a los chinos y sus aliados norcoreanos al norte del paralelo 38 (Kleiner, 2001). A partir de este momento, la guerra degeneró en un prolongado conflicto de desgaste y estancamiento. Los chinos abandonaron sus tácticas anteriores y practicaron la guerra de guerrillas clásica, lo que resultó en miles de bajas de los soldados bajo el Comando de la ONU (Wright, 2001).

Ante el hecho de que los Estados Unidos intervinieron en Corea, la élite política de China interpretó que el momento del primer enfrentamiento con los americanos había llegado (Kleiner, 2001). Para Mao, la liberación de Taiwán y la conquista del Tíbet eran tareas urgentes de la nueva república. Por lo tanto, si China quería alcanzar esas metas tarde o temprano tendría que derrotar a los Estados Unidos. Además, Mao pensaba que para entonces China no podía luchar exitosamente en el mar como lo hacía el ejército norteamericano. En consecuencia, si se debía enfrentar a Estados Unidos, Corea sería un mejor lugar para hacerlo. Por lo tanto, la Guerra de Corea se presentó como la mejor opción (Lee, 2001).

Sin embargo, enfrentar a los Estados Unidos en combate era una tarea muy complicada debido a la enorme disparidad en la capacidad militar entre la República Popular China y los Estados Unidos. Los planes de desmovilización del ejército chino seguían dejando al ejército chino con aproximadamente tres millones de soldados, pero estas tropas estaban mal equipadas, viajaban a pie y carecían de apoyo aéreo y naval (Kennedy, 2001). Además, le faltaba un robusto sistema logístico para abastecer a las tropas. Durante la revolución, las fuerzas comunistas se basaron en gran medida en los lugareños simpatizantes para apoyos en las labores de inteligencia, la obtención de suministros básicos y el ocultamiento cuando era preciso. Sin embargo, en Corea, los soldados chinos serían extranjeros, lo que dificultaría enormemente la comunicación y la 
cooperación con la población local. El simple hecho de abastecer a las fuerzas chinas en Corea era un desafío formidable (Kennedy, 2001).

Youjun (2015) ha reconstruido el dilema al interior de la cúpula del gobierno chino respecto a intervenir o no en la Guerra de Corea. Para entonces, después de más de una década de guerras, China se estaba reconstruyendo. La inquietud de la élite política era si China podría resistir otra guerra, esta vez contra la mayor potencia militar del mundo. Pero, por otra parte, si China se mantenía al margen, las fuerzas estadounidenses, el ejército de la República de Corea y el Comando de las Naciones Unidas ciertamente derrotarían a Corea del Norte, lo que la postre podría conllevar más desastres para China. Aún más, al utilizar la Guerra de Corea como pretexto, los Estados Unidos habían enviado una presencia aérea y marítima al estrecho de Taiwán lo cual fue visto como una violación de la soberanía china y una seria amenaza para su futuro. Estos dilemas envolvieron las discusiones de los líderes Liu Shaoqi, Zhou Enlai, Zhu De, Peng Dehuai, Lin Biao y, por supuesto, Mao Zedong.

Yojun (2015) afirma que, en los últimos días de septiembre de 1950, en una reunión ampliada del Buró Político del Comité Central del PCCh, Lin Biao se mostró cauteloso y mantuvo una actitud negativa hacia la guerra. Lin enumeró las cifras del armamento chino y estadounidense para demostrar la gran disparidad de fuerza entre los dos países y señaló que las consecuencias serían inimaginables si China enviara tropas a Corea. Casi todos los demás líderes se mostraron escépticos o se opusieron a una intervención. Mao convocó a otra reunión ampliada del Buró Político para el día 4 y 5 de octubre. El mariscal Peng Dehuai, quien no había estado presente en la reunión anterior, asistió esa vez. Para él, enviar tropas a Corea del Norte era esencial; si las tropas estadunidenses se estacionaban en el río Yalu y Taiwán, Estados Unidos podría encontrar una excusa en cualquier momento para declarar la guerra a China.

Conforme a Youjun (2015), después de escuchar las opiniones de los participantes, Mao sentenció: "China y Corea del Norte son países socialistas bajo el liderazgo del partido comunista. Deberíamos estar listos para venir al rescate de los que están en peligro, sobre todo de nuestro vecino amigo Corea del Norte, con el que estamos tan conectados geográfica y políticamente. ¿Cómo podríamos ser espectadores? Debemos luchar en esta guerra precisamente para evitar que China se convierta en la próxima víctima de la agresión. ¡No hay alternativa!” Finalmente se alcanzó un consenso: China enviaría tropas expedicionarias para ayudar a Corea del Norte a resistir la agresión de los Estados Unidos como una medida preventiva para proteger su propio territorio. 
Ahora bien, como lo ha advertido Hudson (2013), el análisis de la toma de decisiones en la política exterior -que incluye, evidentemente ir o no a una guerra- en realidad es el resultado de una constelación de decisiones que son asumidas como momentos intensos de compromiso en un proceso perpetuo de acción, reacción y más acción en múltiples niveles que involucran a una variedad de actores. Desde esta perspectiva, el constructivismo nos propone que la identidad y no solamente los intereses racionales y calculados, actúan como telón de fondo en la toma de decisiones. En el caso de la intervención de China en la Guerra de Corea sugiero que la identidad enmarcó la decisión de participar en el conflicto armado de tres maneras concretas.

En primer lugar, la identidad de un Estado humillado que fue despojado de su riqueza y del lugar preponderante que había ocupado en el Este de Asia, el cual eventualmente debería ser restituido. Corea, había estado bajo la tutela china. La imposibilidad de continuar con esa tutela se debió, desde la narrativa china, a las guerras imperialistas que este país sufrió en el siglo XIX. En consecuencia, entrar en este conflicto era restituir en cierta manera el rol de hermano mayor que velaba por el bienestar y prosperidad de los hermanos menores que había funcionado como discurso legitimador del sistema tributario que dominó las relaciones interestatales en Asia Oriental hasta principios del siglo XIX. Este sistema es el que Fairbanks (1969) postuló como un "orden mundial jerárquico sino-céntrico", en el que China tenía una relación señor-vasallo en términos verticales con las unidades políticas vecinas inspirada por los valores confucianos.

En segundo lugar, la idea del Estado chino como vanguardia del movimiento comunista derivada de la voluntad del pueblo chino para alcanzar las metas más altas sin importar las condiciones materiales concretas. Esta visión se desprende de la interpretación de Mao y algunos líderes del PCCh que apuntaban que, a pesar de que conforme a Marx el desarrollo histórico dependía de la superación de las contradicciones del mundo material, la voluntad podía acelerar las fuerzas históricas a través de una voluntad férrea, sin esperar que existieran condiciones materiales objetivas para las grandes transformaciones (Chan, 2003). En este sentido, si en verdad la voluntad era suficiente para alcanzar las metas propuestas y el Estado chino se distinguía por ser tenaz y perseverante, el no entrar a la guerra sería una contradicción. Por lo tanto, la voluntad de los "voluntarios" chinos que participarían en la guerra era suficiente para derrotar a un ejército superior tecnológicamente como era el estadunidense. Este hecho podría explicar por qué Mao conside- 
raba inevitable la entrada de China a la guerra en ese momento en particular, más allá del cálculo racional de que algún día China tendría que enfrentar las fuerzas imperialistas en cualquier otro lugar.

Finalmente, se encuentra la identidad solidaria del Estado chino frente a las dificultades y vicisitudes que otros Estados comunistas, como Corea, enfrentaban por oponerse a las fuerzas imperialistas. Así, la posición de Peng Dehuai en la segunda reunión del Buró Político del Comité Central demuestra una lógica de toma de decisiones basada en la nueva identidad de China como miembro del mundo socialista, es decir, como una manera de identificación y solidaridad con los aliados comunistas, a pesar del enorme costo que eventualmente tuvo esta decisión y que se analizan en la siguiente sección.

\section{Las consecuencias de la Guerra de Corea para China}

Los chinos y los norcoreanos sufrieron el mayor número de víctimas en la guerra. Las estimaciones varían, pero se ha sugerido que más de 2 millones de civiles norcoreanos murieron y cerca de 500,000 soldados norcoreanos. Las estimaciones más bajas para el número de soldados chinos muertos son alrededor de medio millón, con una cifra máxima de 3 millones. Alrededor de un millón de civiles surcoreanos fueron asesinados; 415,000 tropas sudcoreanas fallecieron y 429,000 personas fueron heridas. Las muertes estadounidenses se han calculado en 54,246, de los cuales 33,629 fueron en combate, mientras que 105,785 soldados resultaron lesionados (Simons, 1995).

A pesar de su costosa participación en la guerra, China pudo mejorar su estatus como una nación revolucionaria en el contexto internacional y se unió a los Estados Unidos y la Unión Soviética para asumir un papel legítimo en la gestión de los asuntos coreanos. Los chinos participaron en la Comisión de Armisticio Militar en Panmunjom que sentó las bases para el armisticio general que dio fin a las hostilidades y que continua vigente hasta el día de hoy (Leitich, 2001). Además, una comisión china asistió a la Conferencia de Ginebra sobre Corea e Indochina en 1954 (Leitich, 2001). Asimismo, los chinos mantuvieron su presencia militar en Corea del Norte hasta 1958 (Lee, 2001).

La participación en la Guerra de Corea fortaleció el liderazgo de los comunistas al interior de China. Por una parte, permitió mantener un discurso de que la amenaza de la guerra era real y que las potencias imperialistas podrían hacer cualquier cosa para agredir a China algún día. Por lo tanto, la lealtad al régimen era también una cuestión de seguridad nacional que no podía ser 
ignorada. Además, la población en China vio por primera vez en más de un siglo que su país enfrentaba con éxito a la mayor potencia militar del mundo y no salía derrotada, aunque esto fuera una verdad a medias. Por último, este evento terminó por consolidar el liderazgo de Mao al interior del Partido Comunista durante los primeros años de vida de la República Popular. En este sentido, Mao utilizó la idea de victoria para impulsar la movilización política del pueblo chino, desatando una nueva ola de nacionalismo revolucionario en la población (Chen, 2001).

Por su parte, al concluir la guerra, el gobierno estadunidense adoptó una línea más dura de contención militar, así como de aislamiento diplomático y sanciones económicas contra Corea del Norte y China (Lee, 2001). Esta fue una de las razones principales por las cuales China se mantuvo aislada de la comunidad internacional en su reincorporación a las Naciones Unidas en el año de 1971 (Wright, 2001). Además, como lo ha advertido Kennedy (2011), la presencia militar estadunidense en Corea y el Pacífico asiático frustraron los planes de la élite política china de recuperar Taiwán, una situación que se consideraba inadmisible y que continúa hasta el día de hoy.

Después de la Guerra de Corea, China ajustó rápidamente su posición en asuntos internacionales y se colocó al centro de las confrontaciones ideológicas y militares entre los dos campos contendientes, encabezados por la Unión Soviética y los Estados Unidos. El papel activo que China jugó en el Este de Asia convirtió este campo de batalla de la Guerra Fría en un extraño "buffer" entre Moscú y Washington porque, con China, era menos probable que la Unión Soviética y los Estados Unidos se vieran involucrados en una confrontación militar directa (Sun \& Li, 2014).

La guerra cambió la política exterior de China en tres rumbos. En cuanto a las capacidades materiales, resultó claro para la dirigencia china que habían logrado detener el avance de las fuerzas estadunidenses y sudcoreanas gracias al enorme costo en vidas humanas que esto había significado. Además, el resultado de la guerra fue un empate sin un ganador claro. Por lo tanto, si China quería consolidar su seguridad nacional y eventualmente convertirse en un líder de los países en desarrollo, necesitaba incrementar sus capacidades materiales. Este factor favoreció la idea de avanzar velozmente hacia la industrialización del país y dio paso al desastroso programa del Gran Salto hacia Adelante, que inició en 1958, cuya meta era que China alcanzara la capacidad de producción de acero equivalente a la del Reino Unido en un periodo de quince años (Lawrence, 2002). Asimismo, el gobierno chino apostó por 
consolidar su capacidad militar cuyo logro más notable fue el desarrollo de la bomba atómica en 1964, una vez que la Unión Soviética rechazó la petición de proporcionar este tipo de armamento (Manson, 1996). China se unió así al selecto grupo de naciones en poseer un arma atómica colocándolo en una situación distinta en el escenario internacional en cuanto a la amenaza que representaba por su capacidad militar.

En cuanto a la proyección internacional, China emergió del conflicto con las bases para reconfigurar su identidad, autoproclamándose líder del movimiento de los países no alineados, denunciando cualquier forma de imperialismo, ya fuera el imperialismo estadunidense o, más tarde, el soviético, teniendo como punto de partida el rol protagónico desempeñado en la Conferencia de Bandung en 1955 (Jian, 2008). Asimismo, se construyó la idea de que China se apartaba de las visiones revisionistas del socialismo soviético lo que eventualmente llevó al enfriamiento de las relaciones con la Unión Soviética a partir de 1956 y la división del bloque soviético (Wright, 2001). En este sentido, China estableció sus principios de la política exterior bajo estas premisas, las que se convirtieron en el marco normativo de su interacción con los demás Estados. Además, en China ideológicamente se consolidó una nueva forma de marxismo, el maoísmo, que influyó en la formación de guerrillas que apoyaron los movimientos de liberación nacional. En la percepción de las guerrillas comunistas del mundo, si la Unión Soviética era el fundador del régimen norcoreano, China era su salvador. Esto condujo a la postre también a la Revolución Cultural en China como la última jugada maestra de Mao para dirigir el rumbo de China a un nuevo amanecer totalmente distinto a la doctrina del comunismo primogénito de la Unión Soviética (Lawrence, 2012).

En lo que respecta a los intereses en el contexto internacional, China consolidó una política exterior que le permitiera garantizar el control total de su territorio. Por una parte, el gobierno chino tuvo éxito en cuanto al Tíbet que invadió en 1961. Sin embargo, la tarea pendiente fue la isla de Taiwán, la cual se mantiene hasta el día de hoy como un Estado independiente (Phillips, 1996).

\section{Conclusiones}

La decisión de la élite política china de enviar tropas a la Guerra de Corea ha sido ampliamente debatida. Como lo ha apuntado Li (2014), la mayoría de los historiadores chinos argumentan que Mao hizo una decisión racional, correcta y necesaria; la intervención de China aseguró sus fronteras del no- 
reste, fortaleció las relaciones chino-soviéticas y salvó al régimen norcoreano. Debido al involucramiento militar en el conflicto a una gran escala, China actuó como un importante actor por primera vez desde la Primera Guerra del Opio entre 1839 y 1842. Sin embargo, algunos historiadores en China y en Estados Unidos desafían esta interpretación y condenan a Mao por su mal juicio que costó la vida de cientos de miles de soldados chinos. Algunos más asumen una posición intermedia y argumentan que Mao tenía pocas alternativas políticas en su esfuerzo por lograr la plena aceptación en el mundo comunista a comienzo de la década de 1950 (Li, 2014).

Las interpretaciones de la lógica detrás de la decisión de China de entrar en el conflicto coreano han pasado de enfatizar la renuencia y cautela del gobierno chino a enfatizar el entusiasmo y la imprudencia de los líderes encabezados por Mao. Hasta fines de la década de 1980, los estudios sobre la intervención china en Corea destacaron los intentos del PCCh de disuasión y escalada progresiva que llevaron finalmente a los Voluntarios del Pueblo Chino a cruzar el río Yalu. Sin embargo, en un marcado contraste, estudios publicados a partir de la década de 1990, subrayan el impulso de Beijing para perseguir el conflicto armado (Scobell, 2004).

Por otra parte, solo meses después de la nueva alianza chino-soviética, el hecho de que China enviara o no sus tropas para ayudar a Corea, se convirtió en el tema más importante entre Stalin y Mao y esta decisión fue la primera gran prueba de esa alianza en la que muchas consideraciones sutiles entraron en juego entre los dos líderes (Zhihua, 2012). De hecho, se ha asumido que China se encontraba en ese momento vulnerable ante las presiones de Stalin, razón por la cual los líderes chinos tuvieron que aceptar sus condiciones para recibir la ayuda que les permitiera reactivar la economía y en consecuencia terminaron por alinearse con la URSS porque no tenían otra opción.

Sin embargo, el constructivismo ofrece una visión distinta que explica las razones por las cuales la dirigencia de China apoyó una intervención militar en Corea a pesar de los riesgos y costos que dicha decisión representaba. En primer lugar, es preciso señalar que la cúpula del poder en China que asumió esta decisión estaba enmarcada en un contexto histórico y social particular de la historia de China. Por lo tanto, sus paradigmas de lo que China debería ser, más allá de las limitaciones materiales y los riesgos que una decisión de ir a la guerra implicaba cuando la nación estaba exhausta por más de quince años de combates en su territorio, estuvieron enmarcados por estructuras normativas. Estas estructuras se forjaron a partir de una interpretación con- 
creta de la historia de China y del rol que este país debía desempeñar. Esta interpretación fue resultado de la manera en que se imaginó la identidad del Estado chino e influyó en los intereses internacionales de la nueva China.

Es posible afirmar que, para los líderes de China, encabezados por Mao, el peso de "humillación" que sufrió China durante los años previos al triunfo del comunismo demostraba la ambición imperialista de las potencias europeas cuya raíz se encontraba en el sistema capitalista depredador; es decir, se trataba de una lectura de la historia asociada a una interpretación sobre las identidades de otros Estados que determinaban sus acciones.

El intervenir en la guerra tenía un doble propósito, avanzar en la ruta del comunismo como camino de liberación y recuperar el lugar que un día China tuvo en la historia y que violentamente le fue arrebatado. Estas aspiraciones estaban sólidamente vinculadas más a la identidad del Estado chino que a intereses materiales. En este sentido, la identidad de China era producto de una interpretación socialmente construida sobre lo que significaba China como nación. Esta interpretación de la identidad fue una pieza clave al momento de decir intervenir. Aún más, esta identidad marcaba también el deber de China en el nuevo escenario de posguerra de ayudar a otros países en sus propias luchas de liberación en la medida en que China se proclamaba paulatinamente como líder de una tercera vía, más allá de las formas del capitalismo imperialista y del comunismo revisionista.

Los intereses de China detrás de este conflicto consistían en recuperar el prestigio que una vez este país tuvo, lo que incluía el garantizar su integridad territorial y mantener sus áreas de influencia. Pero, como la identidad no es un elemento estático sino en constante transformación, el salir victoriosa del conflicto -al impedir que Estados Unidos y sus aliados capitalistas ocuparan toda la península coreana- le permitió dar un giro a su identidad no dejándola estancada sólo como un país víctima de la ambición imperialista sino identificarse como la vanguardia auténtica de las nuevas formas sociales que darían paso a la etapa más avanzada de la humanidad en una versión de comunismo más auténtico.

Finalmente, el caso de la intervención de China en la Guerra de Corea demuestra que las estructuras materiales y los intereses que se derivan de estas no son suficientes para explicar el comportamiento de los Estados. En este caso, el constructivismo nos sugiere una visión más amplia sobre los factores que determinaron el involucramiento de China en el conflicto. De esta manera, al incorporar el peso que las identidades de los actores y las 
percepciones en torno a estas, nos permiten generar una explicación en el que ir o no a la guerra, con las consecuencias que eso representaba para China en el contexto particular en que se encontraba el gobierno, se vuelve menos mecanicista y menos reduccionista a los factores materiales. Por lo tanto, al introducir el enfoque teórico constructivista en la comprensión de China y la Guerra de Corea en el contexto de la Guerra Fría, es posible asumir una explicación más en sintonía con la complejidad del ser humano y los factores que están detrás de su toma de decisiones.

\section{Referencias}

Adler, E. (2002). Constructivism and International Relations. En W. Carlsnaes, T. Risse \& B. Simmons, (Eds.), Handbook of International Relations (pp. 95-118). Londres: Sage Publications.

Armstrong, C. (2013). Tyranny of the Weak: North Korea and the World, 19501992. Ithaca, Nueva York: Cornell University Press.

Beal, T. (2011). Crisis in Korea: America, China and the Risk of War. Londres: Pluto Press.

Blomstedt, L. (2016). Truman, Congress, and Korea: The Politics of America's First Undeclared War. Lexington, KY: The University Press of Kentucky.

Buckley, R. (2007). The United States in the Asia-Pacific since 1945. Cambridge: Cambridge University Press.

Chan, A. (2003). Chinese Marxism. Nueva York: Continuum.

Chan, R. K. (2018). Korea-China Relations in History and Contemporary Implications. Nueva York: Palgrave McMillan.

Chen, J. (2001). Mao's China and the Cold War. Chapel Hill, NC: the University of North Carolina Press.

Chernoff, F. (2007). Theory and Metatheory in International Relations: concepts and contending accounts. Nueva York: Palgrave MacMillan.

Chu, Y., \& Lin, C. (2003). Consolidating Taiwan's New Democracy amid Competing National Identities. En L. Fernández-Stembridge \& T. Fisac (Ed.), China Today: Economic Reforms, Social Cohesion and Collective Identities (pp. 240-267). Londres: Routledge Curzon.

Fairbank, J. K. (1969). China's Foreign Policy in Historical Perspective, Foreign Affairs, 47(3): 449-463.

Genest, M. A. (2004). Conflict and Cooperation: evolving theories of International Relations. Belmont, California: Wadsworth. 
Griffiths, M., O'Callaghan, T., \& Roach, S. (2008). International Relations: The Key Concepts. Nueva York: Routledge.

Hexiu, Q. (2015). An Attempt at Analyzing the Cross and Triangular Divided Relations among the Four Regimes in China and the Korean Peninsula after World War II. En C. P. Freeman (Ed.), China and North Korea Strategic and Policy Perspectives from a Changing China (pp. 139-154). Nueva York: Palgrave McMillan.

Hobson, J. (2000). The State and the International Relations. Cambridge: Cambridge University Press.

Hudson, V. M. (2013). Foreign policy Analysis: Classic and Contemporary Theory. Maryland: Rowman \& Littlefield Publishers.

Hopf, T. (1998). The Promise of Constructivism in International Relations Theory. International Security, 23(1), 171-200. Recuperado de https://pdfs. semanticscholar.org/9f48/cf16518d285bf30e2c1bd07c9d900a2a8d72. pdf

Hughes, G. \& Dockrill, S.R. (2006). Introduction: The Cold War as a History. En S. R. Dockrill \& G. H. (Eds.), Palgrave Advances in Cold War History (pp. 1-18). Nueva York: Palgrave McMillan.

Jackson, R. \& Sorensen, G. (2003). Introduction to International Relations: Theories and Approaches. Oxford: Oxford University Press.

Jian, C (2008). China and the Bandung Conference: Changing Perceptions and Representations. En S. Tan \& A. Acharya (Eds.). Bandung Revisited: The Legacy of the 1955 Asian-African Conference for International Order (pp. 132-159). Singapur, Nuss Press.

Kaufman, J. P. (2013). Introduction to International Relations: Theory and Practice. Lanham, Maryland: Rowman \& Littlefield Publishers.

Kawamura, N. (2015). Emperor Hirohito and the Pacific War. Washington: University of Washington Press.

Kennedy, A. (2011). The International Ambitions of Mao and Nehru: National Efficacy Beliefs and the Making of Foreign Policy. Cambridge: Cambridge University Press.

Kim, J. (2012). A History of Korea: From Land of the Morning Calm to States in Conflict. St. Bloomington, IN: Indiana University Press.

Kleiner, J. (2001). Korea: A Century of Change. Singapur: World Scientific Publishing.

Kowert, P. (1998). Agent versus Structure in the Construction of National Identity. En V. Kublákova, N. Onuf \& P. Kowert (Eds.), International 
Relations in a Constructed World (pp. 101-122). Armonk, Nueva York: M. E. Sharpe.

Krieger, W. (2006). The International System. En S. R. Dockrill \& G. H. (Eds.), Palgrave Advances in Cold War History (pp. 19-51). Nueva York: Palgrave McMillan.

Kwon, S. (2016). A Critical Evaluation on the Cultural Nationalism of the Two

Koreas. The Journal of East Asian Affairs, 30(2), 133-161.

Lawrence, A. (2002). China under the Communism. Londres: Taylor and Francis.

Lee, C. (2001). Conflict and Cooperation: The Pacific Powers and Korea. En N.

Eberstadt \& R. J. Ellings (Eds.), Korea's Future and the Great Powers, (pp. 51-87). Washington: University of Washington Press.

Lee, J. (2006). The Partition of Korea after World War II: A Global History. New York: Palgrave Macmillan.

Li, X. (2014). China's Battle for Korea: The 1951 Spring Offensive. St. Bloomington, IN: Indiana University Press.

Leitich, K.A. (2001). The Korean Peninsula: A Fifty-Year Struggle for Peace and Reconciliation. En S. G. Benson, N. Matuszak, \& M. A. O’Meara (Eds.), History behind the Headlines: The Origins of Conflicts Worldwide (pp. 149156). Detroit, MI: Gale.

Manson, J. (1996). The Cold War: 1945-1991. Londres: Routledge. Men, J. (2007). Changing Ideology in China and Its Impact on Chinese Foreign Policy. En S. Guo \& S. Hua (Eds.), New Dimensions of China Foreign Policy (pp. 16-41). Lanham, Maryland, United States of America: Lexington Books.

Miller, A. L. \& Wich, R. (2011). Becoming Asia: Change and Continuity in Asian International Relations since World War II. Stanford, CA: Stanford University Press.

Murphey, R. (2009). East Asia: A New History. Nueva York: Pearson.

Oberdorfer, D. \& Carlin, R. (2014). The Two Koreas: A Contemporary History. Nueva York: Basic Books.

Onuf, N. (1998). Constructivism: An User Manual. En V. Kublákova, N. Onuf \& P. Kowert (Eds.), International Relations in a Constructed World (pp. 5878). Armonk, Nueva York: M. E. Sharpe.

Painter, D. (1999). The Cold War: An International History. Londres: Routledge. Phillips, A.B. (2007). Constructivism. En M. Griffiths (Ed.), International Relations Theory for the Twenty-First Century (pp. 60-74). Londres: Routledge. 
Phillips, R. (1996). China since 1911. Nueva York: McMillan Education. Reus-Smith, C. (2005). Constructivism. En Scott Burchill, A. Linklater \& R. Devetak (Eds.) Theories of International Relations (pp. 188-210). Nueva York: Palgrave MacMillan.

Robinson, M. E. (2007). Korea's Twentieth-Century Odyssey: A Short History. Honolulu: University of Hawaii Press.

Sandler, S. (1999). The Korean War: An Interpretative History. Londres: Routledge.

Scobell, A. (2004). Soldiers, Statesmen, Strategic Culture, and China's 1950 Intervention in Korea. En Zhao, S. (Ed.) Chinese Foreign Policy: Pragmatism and Strategic Behavior (pp. 65-75). Londres: Routledge.

Seth, M.J. (2010). A Concise History of Modern Korea: From the Late Nineteenth Century to the Present. Maryland, United States of America: Rowman \& Littlefield Publishers.

Simons, G. (1995). Korea: The Search for Sovereignty. Nueva York: Palgrave Macmillan.

Sun, Y. \& Li, X. (2014). Mao Zedong and the CCP: Adaptation, Centralization, and Succession. En X. Li \& X. Tian (Eds.), Evolution of Power: China's Struggle, Survival, and Success (pp. 25-118). Lanham, Maryland: Lexington Books.

Wendt, A. (1992). Anarchy is what States Make of it: The Social Construction of Power Politics. International Organization, 46(2), 391-425.

Wendt, A. (1994). Collective Identity Formation and the International State. The American Political Science Review, 88(2), 384-396.

Wright, D.C. (2001). The History of China. Westport, Connecticut: Greenwood Press.

Youjun, Y. (2015). Socialism in China (1919-1965). Reading, England: Paths International Ltd.

Zhihua, S. (2012). Mao, Stalin and the Korean War Trilateral Communist Relations in the 1950s. Londres: Routledge. 